\title{
Bowel Movement Frequency, Laxative Use, and Mortality From Coronary Heart Disease and Stroke Among Japanese Men and Women: The Japan Collaborative Cohort (JACC) Study
}

\author{
Yasuhiko Kubota ${ }^{1}$, Hiroyasu Iso ${ }^{1}$, and Akiko Tamakoshi ${ }^{2}$, for the JACC Study Group \\ ${ }^{1}$ Public Health, Department of Social Medicine, Osaka University Graduate School of Medicine, Suita, Osaka, Japan \\ ${ }^{2}$ Department of Public Health, Hokkaido University Graduate School of Medicine, Sapporo, Japan \\ Received May 3, 2015; accepted July 27, 2015; released online December 26, 2015
}

Copyright $(\mathcal{C} 2015$ Yasuhiko Kubota et al. This is an open access article distributed under the terms of Creative Commons Attribution License, which permits unrestricted use, distribution, and reproduction in any medium, provided the original author and source are credited.

\begin{abstract}
Background: The associations of bowel movement frequency and laxative use with cardiovascular disease (CVD) are unclear.

Methods: A total of 72014 subjects (29668 men and 42346 women) aged 40 to 79 years, without a history of CVD or cancer, completed a lifestyle questionnaire at baseline between 1988 and 1990 that included information on bowel movement frequency (daily, every 2-3 days, or once every 4 or more days) and laxative use (yes or no), and were followed-up until 2009.

Results: During the subjects' 1165569 person-years of follow-up, we documented 977 deaths from coronary heart disease (561 men and 416 women), 2024 from total stroke (1028 men and 996 women), 1127 from ischemic stroke (606 men and 521 women), and 828 from hemorrhagic stroke (388 men and 440 women). The prevalence of CVD risk factors, such as diabetes, stress, depression, and physical inactivity, was higher in laxative users and in those with a lower frequency of bowel movements. The multivariable HRs ( $95 \%$ confidence intervals [CIs]) of laxative users were as follows: $1.56(95 \% \mathrm{CI}, 1.21-2.03)$ for coronary heart disease and $1.37(95 \% \mathrm{CI}, 1.07-1.76)$ for ischemic stroke in men, and 1.27 (95\% CI, 1.08-1.49) for total stroke, and 1.45 (95\% CI, 1.17-1.79) for ischemic stroke in women. Similar results were observed even after the exclusion of deaths that occurred early in the follow-up period. A significant association between bowel movement frequency and mortality from CVD was not observed.

Conclusions: Constipation could be a marker of exposure to CVD risk factors, and laxative use could be a risk factor for mortality from coronary heart disease and ischemic stroke.
\end{abstract}

Key words: bowel movement frequency; laxatives; constipation; mortality; atherosclerosis

\section{INTRODUCTION}

There is growing awareness of a link between the gut and cardiovascular disease (CVD), particularly with respect to the progression of atherosclerosis, ${ }^{1-3}$ so cultivation of a more thorough understanding of this relationship is very important.

Constipation, one of the most common digestive disorders, ${ }^{4}$ is associated with lifestyle factors, such as stress and disturbed dietary habits. As such, constipation is expected to be closely linked to CVD. However, to the best of our knowledge, only one study has examined the association of constipation with $\mathrm{CVD}^{5}$; this study concluded that constipation, rather than being a risk factor for CVD itself, is a marker of exposure to CVD risk factors and increased CVD risk, specifically from atherosclerosis. ${ }^{5}$ This appears to be a reasonable conclusion, since many causes of constipation, such as aging and stress, are also risk factors for CVD. In addition to causing constipation, atherosclerosis could cause atherosclerotic diseases, such as coronary heart disease and stroke. ${ }^{6-8}$ However, this study included only women and used their perceived level of difficulty of having bowel movements, rather than bowel movement frequency, as an index of constipation. Therefore, this study's findings require further confirmation among men and an investigation of other factors related to constipation.

In the present study, we investigated how a low frequency of bowel movements and the use of laxatives, which are characteristics often seen in constipated people, might 
influence mortality from coronary heart disease and stroke among Japanese men and women.

\section{METHODS}

\section{Study population}

The Japan Collaborative Cohort Study for the Evaluation of Cancer Risks (JACC study) was sponsored by the Ministry of Education, Sports, and Science and was initiated between 1988 and 1990 in 45 areas in Japan. A previous report described the details of this survey. ${ }^{9}$ Briefly, participants responded to self-administered questionnaires about their lifestyle and medical history regarding cancer and CVD. A total of 110585 subjects (46395 men and 64190 women) aged 40 to 79 years old participated in JACC study. However, since the questionnaires in 12 of the 45 areas did not include information about the frequency of bowel movements and the use of laxatives, 23524 subjects (10 431 men and 13093 women) in these 12 areas were excluded from the present analysis. After this exclusion, 87061 persons were eligible for participation in the present study. Of these, 76174 participants (88\%; 31487 men and 44687 women) completed questionnaires that included information on the frequency of bowel movements and the use of laxatives. In addition, 4160 subjects (1819 men and 2341 women) who had histories of cancer or CVD were further excluded because of the potential direct impact of these diseases on bowel movement and mortality. Ultimately, 72014 subjects (29 668 men and 42346 women) were included. The present study was approved by the ethics committees of the Osaka University Graduate School of Medicine and the Nagoya University School of Medicine.

\section{Main exposure: frequency of bowel movements and the use of laxatives}

Participants were asked to provide information from the past year concerning the average frequency of their bowel movements and their laxative use (yes or no). Participants were grouped into the following three groups and then compared to examine the association of bowel movement frequency with CVD risks: those having a daily bowel movement (reference), those having a bowel movement every 2-3 days, and those having a bowel movement once every 4 or more days. Nonusers (reference) and users of laxatives were compared to examine the association of laxative use with CVD risk factors.

\section{Potential confounding factors}

Potential confounding factors included age (years), sex (male or female), history of hypertension (yes or no), history of diabetes (yes or no), body mass index (sex-specific quintiles), alcohol intake (never, ex-drinker, or current drinker with an ethanol intake of $1-22,23-45,46-48$, or $\geq 69$ grams per day), smoking status (never, ex-smoker, or current smoker of 1-19 or $\geq 20$ cigarettes per day), depressive symptoms (no symptoms, 1 symptom, or $2-4$ symptoms), ${ }^{10}$ perceived mental stress (low, medium, or high), daily walking time (rarely, $<30,30-60$, or $>60$ minutes per day), participation in sports (rarely, 1-2, 3-4, or $\geq 5$ hours per week), dietary fiber intake (sex-specific quintiles), and menopausal status (preor postmenopausal). Participants were asked about average intake frequency of 40 food items without specifying portion size as follows: almost never, once or twice per month, once or twice per week, 3-4 times per week, and almost everyday. ${ }^{11}$ Intakes of foods and nutrients were calculated by the Japanese food composition table (4th edition), and standard portion sizes were derived from weighted dietary records. ${ }^{11}$ The value of dietary fiber intake obtained by enzymatic-gravimetric methods ${ }^{12}$ were derived from the food composition table. ${ }^{11}$ The intake of dietary fiber was adjusted for energy intake using the nutrient residual model. ${ }^{13}$ In addition, since geographical area has been reported to be associated with constipation and risk of cardiovascular disease, ${ }^{14-16}$ living in urban or rural areas was also included as a potential confounding factor. In this study, we defined cities in Japan (population $\geq 50000$ ) as urban areas and towns or villages (population $<50000$ ) as rural areas.

\section{Mortality surveillance}

Mortality surveillance was systematically performed by reviewing death certificates, which were sent to each public health center, and mortality data were centralized at the Ministry of Health and Welfare. ${ }^{17}$ The underlying causes of death were coded according to the International Classification of Diseases, 10th Revision (ICD-10). Deaths were confidently ascertained from death certificates at public health centers. The follow-up of subjects lasted until the end of 2009, or until death, whichever occurred first; exceptions were made for cases in which the follow-up was terminated in a study area, which occurred in four areas in 1999, four areas in 2003, and two areas in 2008. Follow-up endpoints included coronary heart disease, total stroke, ischemic stroke, and hemorrhagic stroke. Death from coronary heart disease was defined as ICD-10 codes I20 to I25, total stroke as I60-I69, ischemic stroke as I63 or I69.3, and hemorrhagic stroke as I60 to I62 or I69.0 to I69.2.

\section{Statistical analysis}

We calculated sex-specific, age-adjusted prevalence and mean values of potential confounding factors and compared laxative use groups using $\chi^{2}$ tests or $t$-tests. For the three bowel movement frequency groups, we performed a test for trend using linear or logistic regression analysis. The person-years of follow-up from the baseline (1988 to 1990) to each endpoint (death, a move from the community, or the end of follow-up) were also calculated. Sex-specific hazard ratios (HRs) and their 95\% confidence intervals (CIs) were computed for mortality outcomes after adjusting for age and 
Table 1. Age-adjusted baseline characteristics according to bowel movements frequency and use of laxatives

\begin{tabular}{|c|c|c|c|c|c|c|c|}
\hline & \multicolumn{4}{|c|}{ Bowel movements frequency } & \multicolumn{3}{|c|}{ Use of laxatives } \\
\hline & Daily & $\begin{array}{c}\text { Every } \\
2-3 \text { days }\end{array}$ & $\begin{array}{l}\text { Every } 4 \text { or } \\
\text { more days }\end{array}$ & $P$ value & No & Yes & $P$ value \\
\hline \multicolumn{8}{|l|}{ Men } \\
\hline Number at risk & 26346 & 3006 & 316 & - & 27902 & 1766 & - \\
\hline Age, years & 56.5 & 58.2 & 61.9 & $<0.001$ & 56.3 & 63.3 & $<0.001$ \\
\hline Body mass index, $\mathrm{kg} / \mathrm{m}^{2}$ & 22.7 & 22.3 & 22.1 & $<0.001$ & 22.7 & 22.4 & $<0.001$ \\
\hline History of hypertension, $\%$ & 20.1 & 17.2 & 16.9 & $<0.001$ & 19.6 & 22.6 & 0.003 \\
\hline History of diabetes, \% & 6.1 & 9.3 & 10.7 & $<0.001$ & 6.2 & 11.3 & $<0.001$ \\
\hline Ethanol intake, g/day & 34.2 & 30.3 & 29.7 & $<0.001$ & 34.1 & 29.6 & $<0.001$ \\
\hline Current smoker, \% & 54.1 & 55.6 & 63.8 & 0.002 & 54.4 & 52.8 & 0.195 \\
\hline High perceived mental stress, $\%$ & 23.0 & 27.0 & 27.6 & $<0.001$ & 22.9 & 32.5 & $<0.001$ \\
\hline Two or more depressive symptoms, \% & 5.1 & 8.6 & 14.0 & $<0.001$ & 5.3 & 9.4 & $<0.001$ \\
\hline Walking $\geq 1$ hour/day, $\%$ & 50.1 & 43.1 & 34.6 & $<0.001$ & 49.9 & 38.9 & $<0.001$ \\
\hline Sports $\geq 5$ hours/week, $\%$ & 7.3 & 5.5 & 4.1 & $<0.001$ & 7.1 & 6.8 & 0.656 \\
\hline Intake of dietary fiber ${ }^{\mathrm{a}}, \mathrm{g} / \mathrm{d}$ & 12.5 & 12.5 & 12.3 & 0.335 & 12.5 & 12.7 & 0.195 \\
\hline Living in urban area, $\%$ & 38.0 & 36.1 & 34.9 & 0.023 & 37.5 & 41.4 & 0.001 \\
\hline Bowel movement frequency every 4 or more days, $\%$ & - & - & - & - & 6.2 & 80.5 & $<0.001$ \\
\hline Use of laxatives, \% & 4.0 & 19.5 & 44.2 & $<0.001$ & - & - & - \\
\hline Having frequent diarrhea, $\%$ & 19.1 & 16.0 & 17.8 & $<0.001$ & 18.7 & 21.4 & 0.008 \\
\hline \multicolumn{8}{|l|}{ Women } \\
\hline Number at risk & 28738 & 11747 & 1861 & & 36589 & 5757 & \\
\hline Age, years & 57.4 & 56.2 & 56.4 & $<0.001$ & 56.8 & 58.6 & $<0.001$ \\
\hline Body mass index, $\mathrm{kg} / \mathrm{m}^{2}$ & 23.1 & 22.6 & 22.5 & $<0.001$ & 22.9 & 23.1 & 0.005 \\
\hline History of hypertension, $\%$ & 22.5 & 20.3 & 18.2 & $<0.001$ & 21.1 & 25.6 & $<0.001$ \\
\hline History of diabetes, \% & 3.5 & 4.5 & 5.3 & $<0.001$ & 3.6 & 6.0 & $<0.001$ \\
\hline Ethanol intake, g/day & 10.4 & 9.4 & 10.4 & 0.056 & 10.0 & 10.7 & 0.199 \\
\hline Current smoker, \% & 4.9 & 5.2 & 9.7 & $<0.001$ & 4.5 & 9.1 & $<0.001$ \\
\hline High perceived mental stress, $\%$ & 19.5 & 21.9 & 28.0 & $<0.001$ & 19.5 & 27.0 & $<0.001$ \\
\hline Two or more depressive symptoms, $\%$ & 7.1 & 9.3 & 13.8 & $<0.001$ & 7.6 & 10.7 & $<0.001$ \\
\hline Walking $\geq 1$ hour/day, $\%$ & 52.9 & 47.7 & 43.4 & $<0.001$ & 52.1 & 44.4 & $<0.001$ \\
\hline Sports $\geq 5$ hours/week, $\%$ & 4.8 & 3.8 & 2.8 & $<0.001$ & 4.6 & 3.5 & $<0.001$ \\
\hline Intake of dietary fiber ${ }^{\mathrm{a}}, \mathrm{g} / \mathrm{d}$ & 12.4 & 12.0 & 11.7 & $<0.001$ & 12.3 & 12.0 & $<0.001$ \\
\hline Menopausal status, \% & 67.4 & 65.5 & 63.8 & 0.002 & 66.8 & 66.1 & 0.176 \\
\hline Living in urban area, $\%$ & 39.5 & 40.6 & 41.0 & 0.026 & 38.7 & 47.5 & $<0.001$ \\
\hline Bowel movement frequency every 4 or more days, $\%$ & - & - & - & - & 2.6 & 15.6 & $<0.001$ \\
\hline Use of laxatives, \% & 7.7 & 22.6 & 48.1 & $<0.001$ & - & - & - \\
\hline Having frequent diarrhea, $\%$ & 9.8 & 6.8 & 5.1 & $<0.001$ & 9.0 & 6.7 & $<0.001$ \\
\hline
\end{tabular}

${ }^{a}$ Energy-adjusted value by nutrient residual model.

other potential confounding factors using Cox proportional hazard models. The proportional hazard assumption in Cox regression was tested, and no violation was found. The SAS version 9.4 software (SAS Institute Inc., Cary, NC, USA) was used for statistical analyses. All statistical analyses were twotailed, and $P$ values $<0.05$ were considered significant.

\section{RESULTS}

Table 1 lists the baseline characteristics according to bowel movement frequency and the use of laxatives. Compared to men with daily bowel movements or male nonusers of laxatives, those with less frequent bowel movements or those using laxatives tended to be older and leaner, drank less alcohol, and walked less, and were more likely to have a history of diabetes and high perceived mental stress, and have depressive symptoms. Women with less frequent bowel movements or those using laxatives were more likely to have a history of diabetes and high perceived mental stress, be current smokers, and live in urban areas; were less likely to take part in physical activities; and had lower intake of dietary fiber than those with daily bowel movement or female nonusers of laxatives. Both men and women using laxatives were more likely to have a history of hypertension than nonusers. In addition, men using laxatives were more likely to have frequent diarrhea than male nonusers of laxatives; on the other hand, female users of laxatives were less likely to have frequent diarrhea than female nonusers of laxatives.

We documented 4604 deaths during the 1165569 personyears of follow-up for the 72014 subjects (29668 men and 42346 women). Nine hundred and seventy-seven deaths resulted from coronary heart disease (561 men and 416 women), 2024 from total stroke (1028 men and 996 women), 1127 from ischemic stroke (606 men and 521 women), and 828 from hemorrhagic stroke (388 men and 440 women) (Table 2).

Age-adjusted and multivariable HRs were calculated for mortality from coronary heart disease, total stroke, and its subtypes, with respect to bowel movement frequency and 
Table 2. Sex-specific, age-adjusted, and multivariable ${ }^{a}$ hazard ratios and $95 \%$ confidential intervals for cardiovascular mortality according to bowel movements frequency and use of laxatives

\begin{tabular}{|c|c|c|c|c|c|}
\hline & \multicolumn{3}{|c|}{ Bowel movements frequency } & \multicolumn{2}{|c|}{ Use of laxatives } \\
\hline & Daily & Every $2-3$ days & Every 4 or more days & No & Yes \\
\hline \multicolumn{6}{|l|}{ Men } \\
\hline Number at risk & 26346 & 3006 & 316 & 27902 & 1766 \\
\hline Person-years & 421406 & 44831 & 4092 & 447244 & 23085 \\
\hline Coronary heart disease, $n$ & 476 & 75 & 10 & 491 & 70 \\
\hline Age-adjusted HR (95\% Cl) & 1.00 & $1.34(1.05-1.71)$ & $1.62(0.87-3.04)$ & 1.00 & $1.76(1.36-2.27)$ \\
\hline Multivariable HR (95\% Cl) & 1.00 & $1.26(0.99-1.62)$ & $1.51(0.80-2.83)$ & 1.00 & $1.56(1.21-2.03)$ \\
\hline Total stroke, $n$ & 880 & 131 & 17 & 925 & 103 \\
\hline Age-adjusted HR (95\% Cl) & 1.00 & $1.21(1.01-1.46)$ & $1.32(0.82-2.13)$ & 1.00 & $1.21(0.98-1.48)$ \\
\hline Multivariable $\mathrm{HR}(95 \% \mathrm{Cl})$ & 1.00 & $1.16(0.96-1.40)$ & $1.13(0.70-1.83)$ & 1.00 & $1.14(0.92-1.41)$ \\
\hline Ischemic stroke, $n$ & 510 & 83 & 13 & 530 & 76 \\
\hline Age-adjusted HR $(95 \% \mathrm{Cl})$ & 1.00 & $1.28(1.01-1.61)$ & $1.60(0.92-2.79)$ & 1.00 & $1.41(1.10-1.79)$ \\
\hline Multivariable HR $(95 \% \mathrm{Cl})$ & 1.00 & $1.23(0.97-1.56)$ & $1.40(0.80-2.45)$ & 1.00 & $1.37(1.07-1.76)$ \\
\hline Hemorrhagic stroke, $n$ & 341 & 45 & 2 & 367 & 21 \\
\hline Age-adjusted HR $(95 \% \mathrm{Cl})$ & 1.00 & $1.14(0.83-1.56)$ & $0.46(0.11-1.85)$ & 1.00 & $0.73(0.47-1.14)$ \\
\hline Multivariable HR $(95 \% \mathrm{Cl})$ & 1.00 & $1.10(0.80-1.51)$ & $0.39(0.10-1.58)$ & 1.00 & $0.68(0.43-1.07)$ \\
\hline \multicolumn{6}{|l|}{ Women } \\
\hline Number at risk & 28738 & 11747 & 1861 & 36589 & 5757 \\
\hline Person-years & 475197 & 190961 & 29082 & 607670 & 87570 \\
\hline Coronary heart disease, $n$ & 288 & 106 & 22 & 334 & 82 \\
\hline Age-adjusted HR $(95 \% \mathrm{Cl})$ & 1.00 & $1.04(0.83-1.29)$ & $1.40(0.91-2.16)$ & 1.00 & $1.42(1.11-1.81)$ \\
\hline Multivariable HR $(95 \% \mathrm{Cl})$ & 1.00 & $0.98(0.78-1.23)$ & $1.25(0.81-1.94)$ & 1.00 & $1.28(0.99-1.64)$ \\
\hline Total stroke, $n$ & 702 & 248 & 46 & 805 & 191 \\
\hline Age-adjusted HR (95\% Cl) & 1.00 & $0.99(0.86-1.15)$ & $1.19(0.88-1.60)$ & 1.00 & $1.37(1.17-1.61)$ \\
\hline Multivariable $\mathrm{HR}(95 \% \mathrm{Cl})$ & 1.00 & $0.98(0.84-1.13)$ & $1.13(0.84-1.53)$ & 1.00 & $1.27(1.08-1.49)$ \\
\hline Ischemic stroke, $n$ & 363 & 130 & 28 & 404 & 117 \\
\hline Age-adjusted HR (95\% Cl) & 1.00 & $1.00(0.82-1.22)$ & $1.37(0.93-2.01)$ & 1.00 & $1.56(1.27-1.92)$ \\
\hline Multivariable HR (95\% Cl) & 1.00 & $0.98(0.80-1.20)$ & $1.29(0.87-1.90)$ & 1.00 & $1.45(1.17-1.79)$ \\
\hline Hemorrhagic stroke, $n$ & 315 & 110 & 15 & 375 & 65 \\
\hline Age-adjusted HR $(95 \% \mathrm{Cl})$ & 1.00 & $0.96(0.78-1.20)$ & $0.86(0.51-1.45)$ & 1.00 & $1.07(0.82-1.40)$ \\
\hline Multivariable $\mathrm{HR}(95 \% \mathrm{Cl})$ & 1.00 & $0.97(0.78-1.20)$ & $0.85(0.50-1.43)$ & 1.00 & $0.98(0.75-1.28)$ \\
\hline
\end{tabular}

$\mathrm{Cl}$, confidential interval; HR, hazard ratio.

${ }^{a}$ Adjusted for age, history of hypertension, history of diabetes, body mass index, alcohol intake, smoking status, depressive symptoms, perceived mental stress, walking, sports, energy-adjusted dietary fiber intake, living in urban areas and menopausal status for women.

the use of laxatives. Men having a bowel movement once every 2-3 days had a significantly higher risk of ageadjusted mortality from coronary heart disease, total stroke, and ischemic stroke compared to those with a daily bowel movement. However, these associations were no longer statistically significant after adjusting for potential confounding factors.

The use of laxatives was associated with higher risks of age-adjusted mortality from coronary heart disease and ischemic stroke in men and women, as well as from total stroke in women only. Further adjustment attenuated, but did not substantially change, these associations, except for the association of laxative use with mortality from coronary heart disease in women. For men, significant multivariable HRs were obtained for coronary heart disease (HR 1.56; 95\% CI, 1.21-2.03) and ischemic stroke (HR 1.37; 95\% CI, 1.07-1.76). For women, significant multivariable HRs were obtained for total stroke (HR 1.27; 95\% CI, 1.08-1.49) and ischemic stroke (HR 1.45; 95\% CI, 1.17-1.79).

To examine the possibility that a serious condition at baseline might have compelled people to use laxatives (reverse causation), we calculated the multivariable HRs for coronary heart disease, total stroke, and ischemic stroke after excluding deaths that occurred early in the study (Table 3 ). Similar results were observed even after the exclusion of deaths that occurred 1 to 7 years from the baseline.

\section{DISCUSSION}

We obtained two major findings in this prospective cohort study of Japanese men and women. First, those who had less frequent bowel movements or used laxatives had a higher prevalence of several previously identified risk factors for CVD, including diabetes, perceived mental stress, ${ }^{18}$ depression, ${ }^{19}$ and physical inactivity compared to those with more frequent bowel movements or nonusers of laxatives. Second, while bowel movement frequency was not associated with risk of mortality from CVD, laxative users had higher risks of mortality from coronary heart disease (men only), total stroke (women only), and ischemic stroke (men and women) compared to nonusers. To the best of our knowledge, this is the first study to investigate the association of bowel movement frequency or laxative use with mortality from CVD in an Asian population. 
Table 3. Multivariable ${ }^{a}$ hazard ratios and $95 \%$ confidential intervals for cardiovascular mortality according to use of laxatives after excluding deaths occurring within 1 to 7 years from baseline

\begin{tabular}{|c|c|c|c|c|}
\hline \multirow[b]{2}{*}{ Use of laxatives } & \multicolumn{2}{|c|}{ Men } & \multicolumn{2}{|c|}{ Women } \\
\hline & No & Yes & No & Yes \\
\hline Person-years & 447244 & 23085 & 607670 & 87570 \\
\hline Coronary heart disease, $n$ & 491 & 70 & 334 & 82 \\
\hline Multivariable HR (95\% Cl) & 1.00 & $1.56(1.21-2.03)$ & 1.00 & $1.28(0.99-1.64)$ \\
\hline \multirow[t]{2}{*}{ Death within 1 year excluded, $n$} & 476 & 67 & 329 & 80 \\
\hline & 1.00 & $1.58(1.21-2.06)$ & 1.00 & $1.28(0.99-1.64)$ \\
\hline \multirow[t]{2}{*}{ Death within 2 years excluded, $n$} & 466 & 64 & 323 & 79 \\
\hline & 1.00 & $1.56(1.19-2.04)$ & 1.00 & $1.30(1.02-1.68)$ \\
\hline \multirow{2}{*}{ Death within 3 years excluded, $n$} & 458 & 63 & 319 & 78 \\
\hline & 1.00 & $1.58(1.20-2.07)$ & 1.00 & $1.31(1.02-1.68)$ \\
\hline \multirow[t]{2}{*}{ Death within 4 years excluded, $n$} & 446 & 57 & 310 & 76 \\
\hline & 1.00 & $1.48(1.11-1.96)$ & 1.00 & $1.33(1.03-1.72)$ \\
\hline \multirow[t]{2}{*}{ Death within 5 years excluded, $n$} & 433 & 55 & 300 & 72 \\
\hline & 1.00 & $1.48(1.11-1.98)$ & 1.00 & $1.32(1.02-1.72)$ \\
\hline \multirow[t]{2}{*}{ Death within 6 years excluded, $n$} & 414 & 51 & 294 & 67 \\
\hline & 1.00 & $1.48(1.10-2.00)$ & 1.00 & $1.27(0.97-1.66)$ \\
\hline \multirow[t]{2}{*}{ Death within 7 years excluded, $n$} & 395 & 45 & 269 & 62 \\
\hline & 1.00 & $1.43(1.03-1.95)$ & 1.00 & $1.31(0.99-1.73)$ \\
\hline Total stroke, $n$ & 925 & 103 & 805 & 191 \\
\hline Multivariable HR $(95 \% \mathrm{Cl})$ & 1.00 & $1.14(0.92-1.41)$ & 1.00 & $1.27(1.08-1.49)$ \\
\hline \multirow{2}{*}{ Death within 1 year excluded, $n$} & 906 & 98 & 791 & 188 \\
\hline & 1.00 & $1.12(0.90-1.39)$ & 1.00 & $1.28(1.09-1.50)$ \\
\hline \multirow[t]{2}{*}{ Death within 2 years excluded, $n$} & 891 & 95 & 770 & 183 \\
\hline & 1.00 & $1.12(0.89-1.38)$ & 1.00 & $1.29(1.09-1.51)$ \\
\hline \multirow[t]{2}{*}{ Death within 3 years excluded, $n$} & 864 & 92 & 757 & 176 \\
\hline & 1.00 & $1.11(0.89-1.39)$ & 1.00 & $1.27(1.08-1.50)$ \\
\hline \multirow[t]{2}{*}{ Death within 4 years excluded, $n$} & 836 & 89 & 737 & 166 \\
\hline & 1.00 & $1.12(0.89-1.40)$ & 1.00 & $1.24(1.05-1.48)$ \\
\hline \multirow[t]{2}{*}{ Death within 5 years excluded, $n$} & 785 & 86 & 705 & 154 \\
\hline & 1.00 & $1.20(0.95-1.51)$ & 1.00 & $1.23(1.03-1.46)$ \\
\hline \multirow[t]{2}{*}{ Death within 6 years excluded, $n$} & 740 & 83 & 667 & 143 \\
\hline & 1.00 & $1.26(1.00-1.60)$ & 1.00 & $1.22(1.01-1.46)$ \\
\hline \multirow[t]{2}{*}{ Death within 7 years excluded, $n$} & 691 & 71 & 636 & 132 \\
\hline & 1.00 & $1.21(0.94-1.55)$ & 1.00 & $1.20(0.99-1.45)$ \\
\hline Ischemic stroke, $n$ & 530 & 76 & 404 & 117 \\
\hline Multivariable $\mathrm{HR}(95 \% \mathrm{Cl})$ & 1.00 & $1.37(1.07-1.76)$ & 1.00 & $1.45(1.17-1.79)$ \\
\hline \multirow[t]{2}{*}{ Death within 1 year excluded, $n$} & 524 & 74 & 401 & 116 \\
\hline & 1.00 & $1.36(1.06-174)$ & 1.00 & $1.45(1.18-1.79)$ \\
\hline \multirow[t]{2}{*}{ Death within 2 years excluded, $n$} & 519 & 73 & 398 & 115 \\
\hline & 1.00 & $1.36(1.06-1.76)$ & 1.00 & $1.46(1.18-1.80)$ \\
\hline \multirow[t]{2}{*}{ Death within 3 years excluded, $n$} & 506 & 71 & 395 & 111 \\
\hline & 1.00 & $1.37(1.06-1.77)$ & 1.00 & $1.43(1.15-1.77)$ \\
\hline \multirow[t]{2}{*}{ Death within 4 years excluded, $n$} & 495 & 69 & 389 & 107 \\
\hline & 1.00 & $1.37(1.05-1.77)$ & 1.00 & $1.41(1.13-1.75)$ \\
\hline Death within 5 years excluded, $n$ & 470 & 66 & 381 & 101 \\
\hline & 1.00 & $1.42(1.09-1.86)$ & 1.00 & $1.37(1.10-1.72)$ \\
\hline Death within 6 years excluded, $n$ & 446 & 66 & 362 & 96 \\
\hline & 1.00 & $1.55(1.19-2.03)$ & 1.00 & $1.40(1.11-1.75)$ \\
\hline Death within 7 years excluded, $n$ & 423 & 58 & 351 & 89 \\
\hline & 1.00 & $1.50(1.13-1.99)$ & 1.00 & $1.35(1.07-1.71)$ \\
\hline
\end{tabular}

$\mathrm{Cl}$, confidential interval; HR, hazard ratio.

${ }^{a}$ Adjusted for age, history of hypertension, history of diabetes, body mass index, alcohol intake, smoking status, depressive symptoms, perceived mental stress, walking, sports, energy-adjusted dietary fiber intake, living in urban areas and menopausal status for women.

People with less frequent bowel movements or those using laxatives were more likely to be exposed to several risk factors for CVD. This result suggests that constipation could be a marker of exposure to CVD risk factors, which is compatible with the conclusions of a previous study. ${ }^{5}$

We found that the use of laxatives was associated with higher risk of mortality from coronary heart disease in men and from ischemic stroke in both men and women, even after adjusting for potential confounding factors. Several mechanisms for these associations could be considered. First, dehydration from the use of laxatives might have led to ischemic diseases because some kinds of laxatives soften defecation by preventing the gut from absorbing water. In fact, men using laxatives were more likely to have frequent 
diarrhea than male nonusers. On the other hand, women using laxatives were less likely to have frequent diarrhea than female nonusers, but this might be due to the possibility that their laxative-softened stools just did not look like diarrhea. Second, one kind of laxative has been reported to induce bacterial overgrowth and inflammation. ${ }^{20}$ The chronic use of this laxative might have destroyed gut flora, leading to chronic inflammation and finally the development of atherosclerosis. Third, serotonin might play a role in the associations observed in this study. Some laxatives increase the formation of serotonin, ${ }^{21}$ which causes vasoconstriction and increases smooth muscle cell aggregation. ${ }^{22,23}$ Therefore, laxatives might have increased the risks of mortality from ischemic diseases through the vasoconstrictive effect of serotonin. Fourth, the use of laxatives might be a marker of autonomic dysfunction and a predictor of cardiovascular disease from autonomic dysfunction. Autonomic dysfunction is associated with several disorders or diseases, including constipation, ${ }^{24}$ depression, ${ }^{25}$ hypertension, ${ }^{26,27}$ diabetes (diabetic neuropathy), and even CVD. ${ }^{28-30}$ In particular, several previous studies suggested that diabetic neuropathy could increase the risk of cardiovascular disease. ${ }^{31}$ Although autonomic nervous function was not measured in this study, we observed that people using laxatives were more likely to have a history of hypertension and diabetes and to have depressive symptoms at baseline, and these individuals had higher risk of mortality from CVD than nonusers. On the other hand, frequency of bowel movement was inversely associated with prevalence of history of hypertension in both men and women. Needing laxatives might reflect severe constipation and autonomic dysfunction more precisely than frequency of bowel movement, and use of laxatives may be a useful tool to predict future CVD events. Finally, since patients with a serious condition are generally more likely to use laxatives than healthier subjects, reverse causation is possible. However, even after the exclusion of deaths occurring within 7 years of the baseline, the association between the use of laxatives and mortality from ischemic diseases remained statistically significant. Therefore, the influence of reverse causation might be negligible.

The strengths of our study include its prospective design, the long follow-up duration, and the large number of participants. Nonetheless, some limitations need to be addressed. First, we had no detailed information on the frequency, amount, or the kind of laxatives used. Further research should use this information to confirm the cause of adverse effects associated with laxatives. Second, bowel movement frequency in this study was self-reported. Therefore, the possibility of misclassification of bowel movement frequency at baseline needs to be considered. Third, mortality data were used as endpoints, which may have led to misclassification of the diagnoses of cardiovascular diseases. However, previous studies have confirmed the validity of using death certificate diagnoses for these outcomes, given that the uses of computed tomography, magnetic resonance imaging, electrocardiography, and cardiac enzyme examination are widespread. ${ }^{32,33}$

In conclusion, constipation could be a marker of exposure to CVD risk factors among Japanese men and women, and the use of laxatives could be a risk factor for mortality from coronary heart disease and ischemic stroke. In clinical settings, constipation might be a useful tool for identifying patients at high risk for CVD, and the careful monitoring of laxative users might be necessary.

\section{ONLINE ONLY MATERIAL}

Abstract in Japanese.

\section{ACKNOWLEDGEMENTS}

We would like to thank all staff members involved in this study for their valuable help in conducting the baseline survey and the follow-up.

Funding sources: This study was supported by the Ministry of Education, Science, Sports, and Culture of Japan (Monbusho) and the Japanese Ministry of Education, Culture, Sports, Science and Technology (Monbukagakusho): grant numbers 1010068, 2151065, 3151064, 4151063, 5151069, 61010076, 62010074, 63010074, 6279102, 11181101, 17015022, 18014011, 20014026, and 20390156.

Conflicts of interest: None declared.

\section{REFERENCES}

1. Sasaki N, Yamashita T, Takeda M, Shinohara M, Nakajima K, Tawa $\mathrm{H}$, et al. Oral anti-CD3 antibody treatment induces regulatory $\mathrm{T}$ cells and inhibits the development of atherosclerosis in mice. Circulation. 2009;120:1996-2005.

2. Wang Z, Klipfell E, Bennett BJ, Koeth R, Levison BS, Dugar B, et al. Gut flora metabolism of phosphatidylcholine promotes cardiovascular disease. Nature. 2011;472:57-63.

3. Tang WH, Wang Z, Levison BS, Koeth RA, Britt EB, Fu X, et al. Intestinal microbial metabolism of phosphatidylcholine and cardiovascular risk. N Engl J Med. 2013;368:1575-84.

4. Bassotti G, Chistolini F, Sietchiping-Nzepa F, de Roberto G, Morelli A, Chiarioni G. Biofeedback for pelvic floor dysfunction in constipation. BMJ. 2004;328:393-6.

5. Salmoirago-Blotcher E, Crawford S, Jackson E, Ockene J, Ockene I. Constipation and risk of cardiovascular disease among postmenopausal women. Am J Med. 2011;124:714-23.

6. Moawad J, McKinsey JF, Wyble CW, Bassiouny HS, Schwartz LB, Gewertz BL. Current results of surgical therapy for chronic mesenteric ischemia. Arch Surg. 1997;132:613-8; discussion 618-9.

7. Silva JA, White CJ, Collins TJ, Jenkins JS, Andry ME, Reilly $\mathrm{JP}$, et al. Endovascular therapy for chronic mesenteric ischemia. J Am Coll Cardiol. 2006;47:944-50.

8. White CJ. Chronic mesenteric ischemia: diagnosis and management. Prog Cardiovasc Dis. 2011;54:36-40. 
9. Tamakoshi A, Ozasa K, Fujino Y, Suzuki K, Sakata K, Mori M, et al. Cohort profile of the Japan Collaborative Cohort Study at final follow-up. J Epidemiol. 2013;23:227-32.

10. Tamakoshi A, Ohno Y; JACC Study Group. Self-reported sleep duration as a predictor of all-cause mortality: results from the JACC study, Japan. Sleep. 2004;27:51-4.

11. Eshak ES, Iso H, Date C, Kikuchi S, Watanabe Y, Wada Y, et al. Dietary fiber intake is associated with reduced risk of mortality from cardiovascular disease among Japanese men and women. J Nutr. 2010;140:1445-53.

12. Prosky L, Asp NG, Schweizer TF, DeVries JW, Furda I. Determination of insoluble, soluble, and total dietary fiber in foods and food products: interlaboratory study. J Assoc Off Anal Chem. 1988;71:1017-23.

13. Willett WC, Howe GR, Kushi LH. Adjustment for total energy intake in epidemiologic studies. Am J Clin Nutr. 1997;65 (4 Suppl):1220S-8S; discussion 1229S-31S.

14. Chu H, Zhong L, Li H, Zhang X, Zhang J, Hou X. Epidemiology characteristics of constipation for general population, pediatric population, and elderly population in China. Gastroenterol Res Pract. 2014;2014:532734.

15. Nishi N, Sugiyama H, Kasagi F, Kodama K, Hayakawa T, Ueda $\mathrm{K}$, et al. Urban-rural difference in stroke mortality from a 19-year cohort study of the Japanese general population: NIPPON DATA80. Soc Sci Med. 2007;65:822-32.

16. Kitamura A, Sato S, Naito Y, Nakagawa Y, Imano H, Ohira T, et al. Trends in the incidence of cardiovascular diseases and risk factors among urban and rural Japanese males. Nihon Koshu Eisei Zasshi. 2001;48:378-94.

17. Kubota Y, Iso H, Ikehara S, Tamakoshi A. Relationship between sleep duration and cause-specific mortality in diabetic men and women based on self-reports. Sleep Biol Rhythms. 2015;13: $85-93$.

18. Iso $\mathrm{H}$, Date $\mathrm{C}$, Yamamoto $\mathrm{A}$, Toyoshima $\mathrm{H}$, Tanabe $\mathrm{N}$, Kikuchi S, et al. Perceived mental stress and mortality from cardiovascular disease among Japanese men and women: the Japan Collaborative Cohort Study for Evaluation of Cancer Risk Sponsored by Monbusho (JACC Study). Circulation. 2002;106: 1229-36.

19. Empana JP, Sykes DH, Luc G, Juhan-Vague I, Arveiler D, Ferrieres J, et al. Contributions of depressive mood and circulating inflammatory markers to coronary heart disease in healthy European men: the Prospective Epidemiological Study of Myocardial Infarction (PRIME). Circulation. 2005;111:
2299-305.

20. Swidsinski A, Ung V, Sydora BC, Loening-Baucke V, Doerffel $\mathrm{Y}$, Verstraelen $\mathrm{H}$, et al. Bacterial overgrowth and inflammation of small intestine after carboxymethylcellulose ingestion in genetically susceptible mice. Inflamm Bowel Dis. 2009;15: 359-64.

21. Capasso F, Mascolo N, Autore G, Romano V. Laxatives and the production of autacoids by rat colon. J Pharm Pharmacol. 1986;38:627-9.

22. Ashton JH, Benedict CR, Fitzgerald C, Raheja S, Taylor A, Campbell WB, et al. Serotonin as a mediator of cyclic flow variations in stenosed canine coronary arteries. Circulation. 1986;73:572-8.

23. Nemecek GM, Coughlin SR, Handley DA, Moskowitz MA. Stimulation of aortic smooth muscle cell mitogenesis by serotonin. Proc Natl Acad Sci USA. 1986;83:674-8.

24. Tougas G. The autonomic nervous system in functional bowel disorders. Gut. 2000;47 Suppl 4: iv78-80; discussion iv87.

25. Kop WJ, Stein PK, Tracy RP, Barzilay JI, Schulz R, Gottdiener JS. Autonomic nervous system dysfunction and inflammation contribute to the increased cardiovascular mortality risk associated with depression. Psychosom Med. 2010;72:626-35.

26. Mancia G, Grassi G, Giannattasio C, Seravalle G. Sympathetic activation in the pathogenesis of hypertension and progression of organ damage. Hypertension. 1999;34:724-8.

27. Grassi G, Mark A, Esler M. The sympathetic nervous system alterations in human hypertension. Circ Res. 2015;116:976-90.

28. Schächinger V, Britten MB, Zeiher AM. Prognostic impact of coronary vasodilator dysfunction on adverse long-term outcome of coronary heart disease. Circulation. 2000;101:1899-906.

29. Goldstein DS, Robertson D, Esler M, Straus SE, Eisenhofer G. Dysautonomias: clinical disorders of the autonomic nervous system. Ann Intern Med. 2002;137:753-63.

30. Hilz MJ, Moeller S, Akhundova A, Marthol H, Pauli E, De Fina P, et al. High NIHSS values predict impairment of cardiovascular autonomic control. Stroke. 2011;42:1528-33.

31. Vinik AI, Ziegler D. Diabetic cardiovascular autonomic neuropathy. Circulation. 2007;115(3):387-97.

32. Kita Y, Okayama A, Ueshima H, Wada M, Nozaki A, Choudhury SR, et al. Stroke incidence and case fatality in Shiga, Japan 1989-1993. Int J Epidemiol. 1999;28:1059-65.

33. Baba S, Ozawa H, Sakai Y, Terao A, Konishi M, Tatara K. Heart disease deaths in a Japanese urban area evaluated by clinical and police records. Circulation. 1994;89:109-15. 